Natur aber schon geändert; sie waren sauer, und setzten Schwefel ab, wie denn der Schwefelwasserstoff von Herrn Deville als das letzte Glied in der Reihe der successiv auftretenden Gasbestandtheile der Fumarolen betrachtet wird.

\title{
John Eliot Howard's Monographie der Chinarinden.
}

$\mathrm{Zu}$ den interessantesten und werthvollsten Erzeugnissen der botanisch-medicinischen Literatur des Jahres 1862 gehört ohne Zẉeifel die schöne Monographie John Eliot Howard's über die von $\mathrm{Pavon}$ und seinen $\mathrm{Be}$ gleitern hinterlassenen, die Gattung Cinchona betreffenden Sammlungen.

Es giebt dies höchst geschmackvoll ausgestattete Werk, welches bei Lovell Reere \& Co. in London unter dem Titel "Illustrations of the nueva Quinologia of Pavon" in Folio erschien von 40 meistens neuen Cinchona-Arten nicht nur die ausführliche systematische Beschreibung und vollständige sehr schön ausgeführte $A b$ bildungen in natürlicher Grösse, sondern ergeht sich in den gründlichsten vergleichenden Untersuchungen ihrer Rinden mit den übrigen bekannten Chinarinden und schildert den anatomischen Bau der auf drei musterhaft bearbeiteten Tafeln auf das Natürlichste wiedergegeben wird.

Auch viele für die Physiologie und Medicin sehr interessante chemische Untersuchungen sind in diesem inhaltreichen Werke niedergelegt. An einer Rinde der Cinchona succirubra Pav., welche sich durch den bedeutenden, bisher nicht beobachteten Alkaloidgehalt von 6,25 Proc. auszeichnete (5,5 Proc. wird seither als Maximum angegeben) machte $\mathrm{H}$ owar d die interessante Entdeckung krystallisirter Alkaloide innerhalb der parenchymatischen Rindenzellen. Die farblosen concentrisch gruppirten Krystalle lösten sich in Alkohol und Aether.

Diese wichtige Beobachtung, so wie verschiedene 
andere Argumente führten Howard zu dem Schlusse, dass der eigentliche Sitz der Alkaloide das par enchymatische Zellgewebe sei, wie dies von Weddel für das Chinin, und nicht die dickwandigen Bastzellen, was von andern Pharmakognosten und Physiologen für die Alkaloide im Allgemeinen angenommen wird.

Auch ist Howard der Meinung, dass das Rindenparenchym nicht nur die Alkaloide aufgespeichert enthalte, sondern dass es dieselben erzeuge; und zwar hält er es nach seinen Beobachtungen für wahrscheinlich, dass der Entstehung der Alkaloide die Bildung der Chinagerbsäure vorausgehe, welche vielleicht durch Wechselwirkung mit ammoniakalischen Verbindungen zu der Erzeugung der organischen Basen Veranlassung gebe; eine Ansicht, die auch de Vry aussprach.

Denn Howard fand die von allen seinen Vorgängern in Untersuchung der Chinarinden seit $R \mathbf{u e z}$ und $\mathrm{Pav}$ on gemachte Erfahrung bestätigt, dass die jüngsten Rinden, neben chinasaurem Kalke, vorwiegend adstringirende Stoffe enthalten; vermuthlich Chinagerbsäure und Chinovasäure. Wabrscheinlich deshalb seien die geringen Spuren von Alkaloiden, welche die jüngsten Chinarinden enthalten, sehr schwierig von Tannin zu reinigen, während aus älteren Rinden derselben Art die organischen Basen leicht $z u$ isoliren sind. Von diesem Verbalten überzeugte sich Howard durch Analysen sehr dünner Zweigrinden-Röhren der C. Calisaya deren etwas dickere, von dem gleichen Standorte gesammelte Stücke von Astrinden eine grössere Menge leicht rein darstellbares Chinin enthielten. In den dicken Platten von Stammrinden waren noch mehr Alkaloide enthalten, weniger in denen der Wurzel.

Folgendes sind die numerischen Resultate der von How ard angestellten Analysen der Rinde der $C$. Calisaya, welche für diese Ansicht zu sprechen scheinen.

Astrinden (canutos) der C. Calisaya enthielten: 
Chinin und etwas Cinchonidin 3,21 Proc.

Cinchonin

$$
\text { Summa ... } \frac{0,01,}{3,22 \text { Proc. }}
$$

Etwas dickere Astrinden (charquesilla) gaben:

Chinin und etwas Cinchonidin 3,41 Proc.

Cinchonin $\ldots \ldots \ldots \ldots \ldots \ldots, \frac{0,03}{n}$,
Summa $\ldots$

Stammrinden (tabla):

Chinin und etwas Cinchonidin 3,55 Proc.

Cinchonin

$$
\text { Summa ... } \frac{0,04 \quad n}{3,59 \text { Proc. }}
$$

Durch die anatomische Untersuchung der analysirten Rinden erkannte Howard, dass mit der gesteigerten Bildung oder Anhäufung von Alkaloiden die Grenze zwischen Bast und Zellgewebe mehr und mehr verschwinde, eine Erscheinung, die ich in meinen nedicinischen Chinarinden Neu-Granadas, pag. 46", durch allmälige und continuirliche Umänderung der secundären Rinde (Bastschicht) in parenchymatisches Zellgewebe, und schliesslich in Kork- und Borkengewebe erklärte, indem alle Elementarorgane des Rindengewebes ihre Function und dem entsprechend ihre Structur ändern.

Eben so wie mit dem Alter der Rinde der Gehalt an organischen Basen zunimmt (eine Thatsache, die schon längst von den Pharmakognosten als Gesetz erkannt wurde), ist auch der Standort der Individuen von Einfluss auf den Gehalt an organischen Basen. Howard führte mehrere Beobachtungen von Spruce und Cordover an, die für die Beständigkeit dieser von mir gemachten Erfahrung sprechen. In tiefer gelegenen, wärmeren Gegenden wurde von diesen Beobachtern die China rubra ärmer an organischen Basen gefunden als in den an höher gelegenen Orten gesammelten Rinden dieselben enthalten waren.

Entsprechend dem grösseren oder geringeren Gehalt an organischen Basen ändert sich auch die Structur- 
beschaffenheit der Rinde, was gleichfalls von Herrn Spruce an Ort und Stelle beobachtet wurde, indem derselbe in tiefer gelegenen Gegenden, die schon ein länger andauerndes trocknes Klima haben, die Rinden der $C$. Calisaya holzig fand, wäbrend dieselbe Pflanzenart in den höheren, während des gạnzen Jahres fast gleichförmig feuchten, regnerischen und nebligen Gegenden eine an Parenchym reichere Rinde hatte.

Es stimmen diese correspondirenden chemischen und physikalischen Eigenschaften der Chinarinden mit den Erfahrungen der Sammler and Händler, welche schon Weddel (Historie naturelle des Quinquinas 1849. pag. 25) anführt und bestätigt, dass der Bruch für die Beurtheilung hinsichtlich des Alkaloidgehaltes dienen könne*): indem diejenigen Rinden, deren Bruch sich dem korkigen nähere mehr Chinin, diejenigen deren Bruch kurzfaserig sei, mehr Cinchonin enthielten.

Wenn Weddel, in Rücksicht auf die Resultate der chemischen Untersuchung verschiedene. Sorten von Chinarinden aus diesen Erscheinungen annehmen zu können glaubt, dass das Cinchonin im Zellgewebe der Aussenrinde, das Chinin aber in den parenchymatischen Zellen der Bastschicht enthalten sei; so stellt Howard mit Recht diesen Folgerungen Weddel's die Bemerkung entgegen, dass es unthunlich sei, ans der chemischen Analyse zweier anatomisch verschiedener von verschiedenen Pflanzenarten abstammenden Rinden auf die chemische Constitution von anatomischen Variationen der Rinde einer dritten Species zu schliessen, da vielmehr jede specifisch eigenthümliche Cinchone auch einen bestimmten aber innerhalb gewisser Grenzen variablen Gehalt an organischen Basen habe.

*) Die von Aen Varietäten der C. lancifolia. Mut. abstammen. den Viarietäten der China flava dura und Ch. flava fibrosa entsprechen gleichfalls diesen Erfahrungen (die medicinischem Chinarinden N. Granadas, pag. 54). Die an organischen Basen reichen Variationen der Ersteren brechen wie Pappe. 
Die Analysen der in Aussen- und Innenrinde gesonderten Rinden verschiedener Cinchonen begründen den Einwurf Howard's und machen es in Bezug anf den letzten Satz Weddel's wahrscheinlich, dass derselbe in umgekehrter Form sich der Wahrheit mehr nähere.

Howard fand in der parench y matösen Aussenrinde einer C. lancifolia Mut., die nur wenig von dem nicht gänzlich zu sondernden Bastgewebe der Innenrinde enthielt:

Chinin ............. 1,18 Proc.

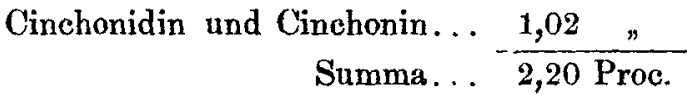

In der Innenrinde dagegen, die nur aus Bastgrewebe bestand, war enthalten:

Chinin................ 0 Proc.

Cinchonidin and Cinchonin...
Summa... $\frac{0,93}{0,93 \text { Proc. }}$

Zugleich widerlegt diese Untersuchung die Ansicht, dass die Alkaloide in den verdickten Bastzellen enthalten seien, wogegen auch die Resultate von Rindenanalysen der C. lancifolia Mut. in verschiedenen Entwickelungsstadien derselben sprechen.

In den dünnen Röhren dieser Cinchona, die zum grossen Theile aus Zellgewebe bestanden, fand nämlich Howard:

Chinin............. 1,07 Proc.

Cinchonin und Cinchonidin... $\frac{0,88 \%}{\text { 1,95 Proc. }}$

Mittlere etwa 3 Linien dicke gerollte Rinden enthielten:

Chinin............. 1,00 Proc.

Cinchonin und Cinchonidin... $\frac{0,90 \text { n }}{\text { Summa... }}$

Starke, fast platte, über 1/2 Zoll dicke Rinden, welche vorwiegend aus Bastgewebe bestanden, gaben: 
Chinin............. 0,71 Proc.

Cinchonin und Cinchonidin... $\frac{1,03 \pi}{1,74 \text { Proc. }}$

Es scheinen diese Resultate mit den oben angeführten Analysen der C.Calisaya im Widerspruche zu stehen, indem hier die älteren Rinden weniger organische Basen entbalten, was aber darin seinen Grund hat, dass von den älteren dieser Handelsrinden die parenchymatöse Aussenschicht durch die Sammler abgekratzt war, wie dies gewöhnlich zu geschehen pflegt (Howard, pag. 5 des anatomischen Abschnittes). (Jene Rinden der C. Calisaya waren speciell für Howard's Untersuchungen, wie es scheint, von Herrn Spruce gesammelt.)

Diese nicht nur in physiologischer, sondern auch in medicinischer und merkantilischer Beziehung sehr beachtenswerthen Ergebnisse werden unterstützt durch eine vergleichende Analyse des äusseren parenchymatösen und des inneren Bastgewebes der China rubra dura von Cinchona succirubra Pav., welche Howard ausführte, indem er ein $1 / 3$ Zoll dickes, 2 Zoll langes und 6 Zoll breites Rindenstück mit deutlichem Harzringe in 2 Theile von gleichem Gewichte zerlegte, in einen äusseren, der aus Zellgewebe mit dem Harzringe und etwas wenigem Baste bestand, und in einen inneren Theil, der vollständig dem Bastgewebe angehörte.

Der äussere Theil war auch hier, wie oben bei der Ch. flava dura, der Hauptsitz des Chinins, so wie des grössten Theiles des Farbstoffes und Gummis. Es ergab diese Analyse den grössten bisher beobachteten Gehalt an organischen Basen, nämlich 8,5 Proc. davon waren:

Reines Chinin ........ 3,65 Proc.

Unreines Chinin ......... 2,66 "

Cinchonidin .......... 1,34,

Cinchonin.............

Ferner enthielt dieses parenchymatische Gewebe bitteres 
Gummi 5 Proc., Chinaroth, wenig chinasauren Kalk, Harz und Chinovasäure.

Die Innenrinde dagegen enthielt nur 5,28 Proc. organische Basen; davon waren:

Cinchonin . ............ 2,0 Proc.

Chinidin, Cinchonidin und Chinin... 3,28n vom Chinin, welches schwierig rein dargestellt werden konnte, waren 0,77 Proc., also vier- bis fünfmal weniger als in der Aussenrinde; Chinovasäure enthielt sie eben so viel als die Aussenrinde und chinasauren Kalk viel mehr als diese.

Das aus allen diesen Versuchen sich ergebende Resultat, dass die verdickten Bastzellen nicht die Behälter der Alkaloide, und insbesondere nicht die Behälter des Chinins seien, ist um so beachtenswerther als gleichzeitig mit den Untersuchungen Howard's von Wigand (Bot. Zeitschr. Mai 1862) Beobachtungen veröffentlicht wurden, welche diesen zu dem entgegengesetzten Schlusse führten.

Den Resultaten der vergleichenden Analysen, auf die Wigand sich stützt, könnte wohl mit Grund der Einwand gemacht werden, dass die Zusammengehörigkeit der Rinden hinsichts des Standortes und der Individualität ausser Acht gelassen war, obgleich doch der Einfluss der Ernährung des Individuums auf den Gehalt seiner Gewebe an Secretionsstoffen bekannt ist: so wie dass selbst individuelle Eigenthümlichkeiten der Pflanze oder Behandlungsweise der Rinde bei gleichem Standorte eine Veränderung im Gehalte der Rinden an organischen Bạsen veranlassen können*).

*) Ich erlaube mir, an die von wir gemachte Beobachtung z11 erinnern, dass die Alkaloide in vegetirenden Rinden, welche nicht durch die Wurzel und durch die Blätter ernährt werden, sich verändern und vermindern, dass sie mitbin Secretionsstoffe (nichtExcrete!) gleich den Proteinverbindungen sind; nur, ihrem Zwecke als Reservenährstoff zu dienen entsprechend, krystallisirt und deshalb weniger lejcht zersetzbar. In Popayan 
Wigand stützt sich ferner auf die Eigenschaft der Chinabastzellen, Farbstoffe aus Lösungen anzuziehen, ein Verhalten, das, seinen interessanten Untersuchungen zufolge, wahrscheinlich von dem Gehalte dieser Bastzellen an Alkaloiden herrühre, und glaubt ferner, bemerkt $z u$ haben, dass die Bastzellen beim Erhitzen kurz vor dem Verkohlen die schöne carminrothe Farbe annehmen, welche einem Theile der Zersetzungsprodycte der erbitzten mit Kohlehydraten gemengten organisehen Basen der Cinchonen eigen ist. (Mir gelang es nicht, diese rothe Farbe an den erhitzten und verkoblenden Bastzellen der rothen Rinde zu erkennen, ich sah sie nur sich bräunen.)

Wenn auch nun die Frage über den Entstehungsort der Chinaalkaloide noch nicht endgültig beantwortet ist, so sind dennoch die umsichtigen und genauen Untersuchungen eines so erfahrenen und vielseitig gründlich unterrichteten Analytikers, wie How ard, von der grössten Bedeutung, und fanden bei der Erörterung dieser Angelegenheit die vollste Beachtung.

Dass die Idee Weddel's über die Vertheilung der beiden in den Chinarinden vorwaltenden organischen Basen in Bezug auf Innen- und Aussenrinde nicht begründet ist, geht unzweifelhaft aus Howard's Analysen hervor.

Die primäre Rinde der Cinchonen fand auch ich gänzlich frei von organischen Basen; diese bilden sich erst mit der Entstehung der secundären Rinde und der gleichzeitig auftretenden Korkbildung. Höchst wahrscheinlich ist der Hauptsitz der Alkaloide die Mittelrinde, in

hörte ich, dass von vielen ungeheuren Cinchonenstämmen, von denen anfangs nur die Oberseite in der Eile abgeschält war, noch nach Jabren, nachdem der Wald in Maisfelder sich verwandelt hatte, auch die Rinde der dann umgewendeten Stämme und Aeste abgeschält wurde; die dsnn ohne $Z$ weifel mit andern in benachbarten Districten gesammelten Rinden neu umgehauener Bäume vermischt in den Handel kam. Die Rinde erhält sich närnlich an dem feuchten Stamme jahrelang lebenskräftig und äusserlich unverändert. 
der die Metamorphose der Elementarorgane vor sich geht. Auch Kork und Borke enthalten Alkaloide, wie man an den carminrothen Dämpfen der Zersetzungsproducte erkennt; interessant würde es sein, die Natur dieser Alkaloide abgesondert $\mathrm{zu}$ studiren.

Mögen durch diese wenigen Andeutungen die Fabrikanten und Chemiker, die Pharmaceuten und Aerzte, die Physiologen und Botaniker auf dies inhaltsreiche Werk aufmerksam gemacht seien; gewiss wird jeder von seinem Inhalte mit Interesse Kenntniss nehmen, niemand dasselbe ohne Belehrung aus der Hand legen.

Möchten sich auch andere Pflanzengattungen solcher - Monographen zu erfreuen haben!

Berlin, in Januar 1863.

Dr. H. Karsten. 